\title{
Collision Detection and Reaction: A Contribution to Safe Physical Human-Robot Interaction
}

\author{
Sami Haddadin, Alin Albu-Schäffer, Alessandro De Luca, and Gerd Hirzinger
}

\begin{abstract}
In the framework of physical Human-Robot Interaction (pHRI), methodologies and experimental tests are presented for the problem of detecting and reacting to collisions between a robot manipulator and a human being. Using a lightweight robot that was especially designed for interactive and cooperative tasks, we show how reactive control strategies can significantly contribute to ensuring safety to the human during physical interaction. Several collision tests were carried out, illustrating the feasibility and effectiveness of the proposed approach. While a subjective "safety" feeling is experienced by users when being able to naturally stop the robot in autonomous motion, a quantitative analysis of different reaction strategies was lacking. In order to compare these strategies on an objective basis, a mechanical verification platform has been built. The proposed collision detection and reactions methods prove to work very reliably and are effective in reducing contact forces far below any level which is dangerous to humans. Evaluations of impacts between robot and human arm or chest up to a maximum robot velocity of $2.7 \mathrm{~m} / \mathrm{s}$ are presented.
\end{abstract}

\section{INTRODUCTION}

Physical human-robot interaction and cooperation has become a topic of increasing importance and of major focus in robotics research. Tasks unifying the workspace of humans and robots, which are most common in service applications and also foreseeable in close future industrial applications, will require safe and dependable robot design and control. Generally, this leads to two main concerns which have to be addressed:

1) Protection of the human body has absolute main priority. Beside preventing collisions by monitoring robot execution with external sensors, we are interested here in reducing the effects of undesired impacts that may anyway occur.

2) Protection of the robotic structure needs also special focus since this directly affects the prospects to effectively react to collisions.

Naturally, an enormous variety of potential threats for both parties exist during close cooperation. Work that has been carried out up to now in the field of physical human-robot interaction was mainly based on simulations [2], whereas in the context of robotics, blunt impacts were first evaluated in [3], [4]. Further aspects concerning safety in human-robot interaction were discussed in [5], [6], [7], [8]. To the best of our knowledge attempts to investigate real world threats via impact tests at standardized crash-test facilities and use the outcome to analyze safety issues during physical humanrobot interaction were carried out up to now only in [1]. In particular, it was shown that even with a perfectly fast physical collision detection and reaction scheme one would not be able to decrease the resulting injury indicators for very rigid head impacts at link inertias similar to one of the

S. Haddadin, A. Albu-Schäffer, and G. Hirzinger are with Institute of Robotics and Mechatronics, DLR - German Aerospace Center, Wessling, Germany sami.haddadin, alin.albu-schaeffer, gerd.hirzingerdalr.de

A. De Luca is with Dipartimento di Informatica e Sistemistica, Univerisità di Roma "La Sapienza", Rome, Italy deluca@dis. uniroma1. it This work has been partially funded by the European Commission's Sixth Framework Programme as part of the projects SMERobot ${ }^{\mathrm{TM}}$ under grant no. 011838 and PHRIENDS under grant no. 045359. Thanks to Bertram Wilberg for his help on the mechanical testbed.

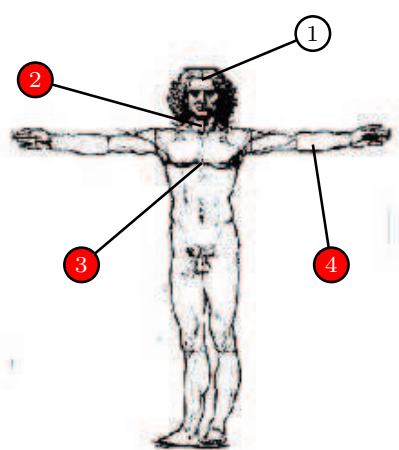

Fig. 1. Investigated human body parts considered in our work. (1) head: A physical collision detection and reaction is generally not able to reduce impact forces for the head, see [1]. Therefore, we will not further discus this issue. (2) neck: The benefit for the neck is shown in Sec. VI-A. (3) this issue. (2) neck: The benefit chest: Experiments described in Sec. VI-E prove the reduction of impact forces for the chest. (4) arm: The arm can be effectively protected as verified in Sec. VI-E.

LWRIII $^{1}$. This is because the investigated hard robot-head impacts last only about $4-10 \mathrm{~ms}$, leading to the observation that one is not able to revert the motors and extract the kinetic energy from the robot within this short impact duration. On the other hand, the analysis of impacts with softer body parts, which are intrinsically slow enough to react on, has to be carried out as well. As a contribution to a future "safety map" this will be one of the main aspects of this paper. The body parts that will be treated here are shown in Fig. 1 (shaded red).

In our previous work in [10] the focus was layed on theory and some preliminary evaluation of the presented collision detection and reaction. In this paper the extension of the theory and an evaluation of the measurable benefit obtained by the collision detection are the topics of interest. The collision detection mechanism proposed in [10], which provides a filtered version of the external collision torque $\tau_{\text {ext }}$, along with improvements concerning detection sensitivity and alternative detection schemes are presented and compared to each other. The signals produced by these torque estimators are used for various collision reaction strategies. In particular, they will serve for a new method of scaling time increments in the trajectory generation, which allows the user to push the robot intuitively forth and back along its desired path even though the robot is still under position control. Together with our collision detection and reaction schemes, this new method gives us the possibility to distinguish between two fundamental types of physical interaction:

- nominal condition: desired physical interaction with the robot;

- fault condition: unexpected collisions, possibly causing injuries.

${ }^{1}$ At the German Aerospace Center (DLR), the DLR Lightweight Robot III (LWRIII) has been developed, which is a robot arm especially characterized by its low weight, a load-to-weight ratio similar to humans, and torque sensing in each joint [9]. 
An appropriate distinction between these cases and an adequate reaction to them is needed to achieve high performance during physical human-robot interaction and to prevent unnecessary interruption during task execution.

All reactive control schemes were implemented and tested on the flexible-joint ${ }^{2}$ robot LWRIII, giving the manipulator the ability to respond in a comfortable fashion to unintended impacts as well as to intended physical cooperation.

To compare the detection and reaction strategies on an objective and repeatable basis, a mechanical verification platform was built which consists of an adjustable 1-DOF mechanical impedance. It is used to point out the major differences between the evaluated reaction schemes and to show their intrinsic drawbacks and limitations as well. The benefit of the presented collision detection and reaction schemes will be illustrated by real impact measurements with a crash-test dummy, a collision test-bed, and a human user. The obtained results indicate that such schemes can play a significant role in ensuring safety to the human operator during physical human-robot interaction. Furthermore, it will be showcased how the collision detection and reaction can help to prevent damage to the robotic structure and thus additionally contribute to an increase in safety due to fault protection.

\section{THE LWRIII: ROBOT AND CONTROLLER}

The LWRIII is a 7-DOF lightweight robot with $1.1 \mathrm{~m}$ reach, flexible joints (due to the use of harmonic drives and joint torque sensors), and was explicitely developed for the direct physical interaction and cooperation with humans. It has a total weight and nominal payload of $14 \mathrm{~kg}$. Furthermore, it is equipped with joint torque sensors in each joint, enabling a direct interaction along the entire robotic structure $^{3}$. For this robot, the following dynamic model is assumed [12]:

$$
\begin{array}{r}
\mathbf{M}(\mathbf{q}) \ddot{\mathbf{q}}+\mathbf{C}(\mathbf{q}, \dot{\mathbf{q}}) \dot{\mathbf{q}}+\mathbf{g}(\mathbf{q})=\tau+\tau_{\text {ext }} \\
\mathbf{B} \ddot{\boldsymbol{\theta}}+\tau=\tau_{m}-\tau_{F} \\
\tau=\mathbf{K}(\boldsymbol{\theta}-\mathbf{q}) .
\end{array}
$$

$\mathbf{M}(\mathbf{q}) \in \Re^{n \times n}$ is the link mass matrix, $\mathbf{C}(\mathbf{q}, \dot{\mathbf{q}}) \in \Re^{n \times n}$ the Centrifugal and Coriolis matrix and $\mathbf{g}(\mathbf{q}) \in \Re^{n}$ the gravitation vector. $\boldsymbol{\theta}$ and $\boldsymbol{q} \in \Re^{n}$ are the measured motor position and the link position of the robot. $\tau$ and $\tau_{\text {ext }} \in \Re^{n}$ are the (elastic) torque at the joints and the torque resulting from external collisions. $\boldsymbol{\tau}_{\boldsymbol{m}} \in \Re^{n}$ and $\boldsymbol{\tau}_{\boldsymbol{F}} \in \Re^{n}$ are the motor torque acting on the motor inertia $\mathbf{B}$ and the friction torque. $\mathbf{K}=\operatorname{diag}\left\{k_{i}\right\} \in \Re^{n \times n}$ is the positive definite joint stiffness matrix.

The measurement of the joint torque $\tau$ allows a unified control framework for position, torque and impedance control for the LWRIII since an additional state measurement is introduced [13]. Switching between these control modes is done within one sampling step (currently $1 \mathrm{~ms}$ ).

\section{Collision Detection}

\section{A. $\hat{\mathbf{r}}$-Observer}

The first collision detection scheme is the disturbance observer introduced in [10], see Fig. 2. In the upper part the rigid body dynamics eq. (1) is sketched and the lower one represents the actual observer. This can also be interpreted as a Hamiltonian observer, since its basic concept is to observe

${ }^{2}$ In this context the flexibility of the joint is caused by the compliance of the lightweight design, the Harmonic Drive gears, and the joint-torque sensors.

${ }^{3}$ For details concerning the full design of the robot, please refer to [9] and [11].

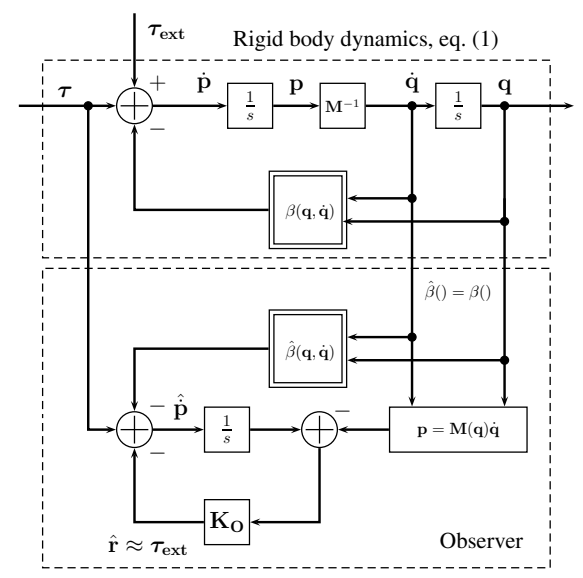

Fig. 2. Block diagram of the disturbance observer, estimating a 1st order filtered version $\hat{\mathbf{r}}$ of the external torque $\tau_{\text {ext }}$. The nonlinear feedback term $\beta(\mathbf{q}, \dot{\mathbf{q}})$ is defined as $\beta(\mathbf{q}, \dot{\mathbf{q}})=\mathbf{C}(\mathbf{q}, \dot{\mathbf{q}}) \dot{\mathbf{q}}+\mathbf{g}(\mathbf{q})-\dot{\mathbf{M}}(\mathbf{q}) \dot{\mathbf{q}}$

the angular momentum $\mathbf{p}=\mathbf{M}(\mathbf{q}) \dot{\mathbf{q}}$, as proposed in [14] and [15]. It can be shown that the observed disturbance is a component-wise filtered version of the real external torque $\tau_{\text {ext }}$ :

$\hat{r}^{i}=\frac{1}{s T_{O}^{i}+1} \tau_{\mathrm{ext}}^{i}=\frac{K_{O}^{i}}{s+K_{O}^{i}} \tau_{\mathrm{ext}}^{i} \approx \tau_{\mathrm{ext}}^{i} \quad \forall i \in\{1, \ldots, n\}$.

$K_{O}^{i}$ can also be interpreted as the filter constant $T_{O}=$ $1 / K_{O}^{i}$ of the $i$ th external joint torque signal component. Furthermore, let $\hat{\mathbf{r}}=\left[\hat{r}_{1} \ldots \hat{r}_{n}\right]^{T}$ be the estimation of the external torque which is used as a collision detection signal, meaning

$$
C D= \begin{cases}1 & \text { if } \exists i: \quad\left|\hat{r}^{i}\right|>\hat{r}_{\text {det }}^{i} \\ 0 & \text { else. }\end{cases}
$$

$\hat{r}_{\text {det }}^{i}>0$ is the collision threshold for the $i$ th axis, which was chosen to be equally $0.1 \tau_{\max }$, i.e. $10 \%$ of the maximum nominal torque of the robot ${ }^{4}$. The main sources of errors limiting the detection threshold for this approach are the model errors and the sensor noise (torque and numerical velocity estimation). By choosing a slow filter constant for $\hat{\mathbf{r}}$ the noise can be reduced at the price of some detection delay.

\section{B. $\hat{\tau}$-Observer}

For a commanded motor trajectory with smooth derivatives of higher order and a well parameterized effective position controller, it can be assumed that

$$
\mathrm{q}_{d} \approx \mathrm{q},
$$

where $\mathbf{q}_{\mathbf{d}} \in \Re^{n}$ is the desired joint position. Thus, $\mathbf{q}$ and its derivatives can be approximated by $\boldsymbol{q}_{\boldsymbol{d}}$ and its derivatives. An estimate of the external joint torque due to collision is given by combining the expected joint torque computed by utilizing (1) in the absence of $\tau_{\text {ext }}=0$

$$
\hat{\tau}\left(\boldsymbol{q}_{\boldsymbol{d}}, \dot{\boldsymbol{q}}_{\boldsymbol{d}}, \ddot{\boldsymbol{q}}_{\boldsymbol{d}}\right)=\hat{\mathbf{M}}\left(\boldsymbol{q}_{\boldsymbol{d}}\right) \ddot{\boldsymbol{q}}_{\boldsymbol{d}}+\hat{\mathbf{C}}\left(\boldsymbol{q}_{\boldsymbol{d}}, \dot{\boldsymbol{q}}_{\boldsymbol{d}}\right) \dot{\boldsymbol{q}}_{\boldsymbol{d}}+\hat{\mathrm{g}}\left(\boldsymbol{q}_{\boldsymbol{d}}\right)
$$

with the measurement of the joint torque $\tau$. This leads to an estimation of the external torque

$$
\hat{\tau}_{\mathrm{ext}}=\tau-\hat{\tau} \approx \tau_{\mathrm{ext}} .
$$

${ }^{4}$ Current implementations allow even a threshold of 3-5\%. 
$\hat{\mathbf{M}}, \hat{\mathbf{C}} \in \Re^{n \times n}$ and $\hat{\mathrm{g}} \in \Re^{n}$ are models of the mass, Coriolis and gravitation terms. Collision detection is based again on (5), except for the substitution of $\hat{\mathbf{r}}$ by $\hat{\tau}_{\text {ext }}$. A major benefit from this approach is that only the joint torque sensor noise is present, which is considerably lower than for velocity. Errors introduced in this case are again the model error as well as the error made by assumption (6). Therefore, this approach performs better for a stiff position controller and pre-planned trajectories with smooth $\mathbf{q}_{\mathbf{d}}$, while for a very soft impedance controller or for non-smooth desired positions (e.g., generated online from a vision system), the detection scheme from Sec. III-A is more advantageous.

\section{Coping with the Robot Model Error}

Both of the above collision detection mechanisms use a collision threshold which should be small enough to allow fast firing of a reaction scheme (sensitivity), but also large enough not to be activated by measurement errors and/or dynamic model errors (false alarms). For the relevant motion velocities, the robot dynamics contain low-frequency signals when compared to the impact torque. One possibility to cope with this robot modelling errors is thus to high-pass filter both detectors (4) and (8). This leads for the $\hat{\mathbf{r}}$-Observer to

$$
\hat{r}_{\mathrm{hpf}}^{i}=T_{O}^{i} s \hat{r}^{i}=\frac{s}{s+K_{O}^{i}} \tau_{\mathrm{ext}}^{i} \quad \forall i \in\{1, \ldots, n\}
$$

if $T_{O}^{i}=1 / K_{O}^{i}$. For the $\hat{\tau}$-Observer this means to use

$$
\hat{\tau}_{\mathrm{ext}}^{\mathrm{hpf}, i}=T_{O}^{i} s\left(\tau^{i}-\hat{\tau}^{i}\right) \quad \forall i \in\{1, \ldots, n\}
$$

as a detector. Of course, this would mean to ignore the very low frequent external torques but they still can be estimated in parallel by (4) or (8). Therefore, for high frequency torque components, i.e. fast rigid impacts, a more sensitive detector is obtained. These versions were both implemented on the LWRIII and allowed to reduce the detection threshold by $50 \%$ in all joints to $0.05 \tau_{\max }$ in comparison to the initial version.

As an alternative to high-pass filtering of the signals, one could use the difference of the two detection schemes as a collision detector. This will remove the model error entirely and the detector happens to be again a high-pass filtered version of $\tau_{\text {ext }}$ :

$$
\underbrace{\tau^{i}-\hat{\tau}^{i}}_{\hat{\tau}_{\mathrm{ext}}^{i}}-\hat{r}^{i} \approx \tau_{\mathrm{ext}}^{i}-\frac{1}{T_{O}^{i} s+1} \tau_{\mathrm{ext}}^{i}=\frac{T_{O}^{i} s}{T_{O}^{i} s+1} \tau_{\mathrm{ext}}^{i}
$$

\section{Collision Reaction Strategies}

As soon as a collision has been detected, a reaction strategy should be activated so as to decrease the potential danger posed to the human. Several strategies were implemented and tested with respect to their effectiveness, especially focusing on their contribution to enhance safety to the human. For a detailed description of these strategies, please refer to [10]. Strategy 0: The robot shows no reaction at all and continues to follow the reference trajectory $\boldsymbol{q}_{\boldsymbol{d}}$. This is used as baseline behavior.

Strategy 1: The robot is stopped as soon as a collision is detected. This is obtained by setting $\boldsymbol{q}_{\boldsymbol{d}}=\boldsymbol{q}\left(t_{c}\right)$, where $t_{c}$ is the instant of collision detection.

Strategy 2: Switch from position control to zero-gravity torque control [13], [16], letting the robot behave in a very compliant way.

Strategy 3: Switch to torque control with gravity compensation but, in contrast to Strategy 2, use joint torque feedback and the signal $\hat{\mathbf{r}}$ to scale down both the motor inertia as well as the link inertia, thus obtaining an even "lighter" robot. Strategy 4: Use the estimated external torque to implement an admittance controller. By defining the desired velocity in the opposite direction of the external torque estimation $\hat{\mathbf{r}}$, the robot "flees" from this disturbance.

\section{TRAJECTORY SCALING}

"Pushing interpolation time back and forth"

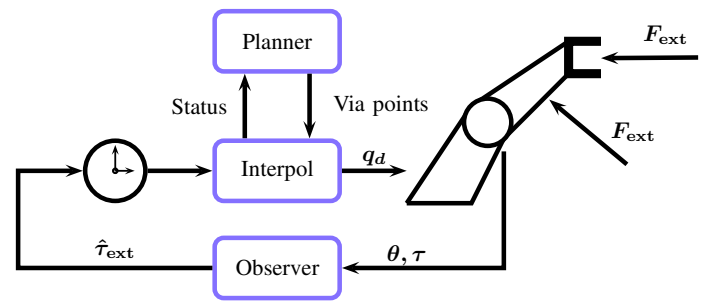

Fig. 3. Idea behind the trajectory scaling: "Pushing interpolation time back and forth". The estimation of the external torque is fed to the time step generator of the interpolator and directly modulates its behavior.

Apart from simply switching the control mode or stopping the robot as soon as a collision was detected, a quite different approach is possible as well, as described hereafter. The idea is to preserve the original motion path and at the same time provide compliant behavior by influencing the time generator of the desired trajectory, see Fig. 3. This scheme can be used to enable a position controlled robot to react compliantly in such a way that it still remains on the nominal path but in case of external disturbances is only able to exert certain maximum forces. Note that this trajectory scaling scheme is driven by the observer output $\hat{\mathbf{r}}$ and can also be combined with any of the previous reaction strategies so as to reduce external torques to zero in case of too dangerous situations.

A desired trajectory is usually parameterized with respect to time, i.e. $\mathbf{q}_{\mathbf{d}}(t) \in \Re^{n}$ in joint space or $\mathbf{x}_{d}(t) \in S E(3)$ in the Cartesian case, whereas we will describe the joint case from now on. For the discrete sampling time $\Delta t$ used in the implementation the current time instant can be written as $t_{i}=t_{i-1}+\Delta t$. If the increment $\Delta t$ is now modified in such a way that it is used to respond to external forces, it can be used to step back and forth along the desired joint path, as a matter of fact by "scaling the trajectory in time", see Fig. 4. This can simply be done by re-defining the interpolation time as

$$
t_{i}:=t_{i-1}+f_{s}\left(\Psi\left(\hat{\mathbf{r}}_{i}\right)\right) \Delta t
$$

In our implementation the trajectory scaling input based on

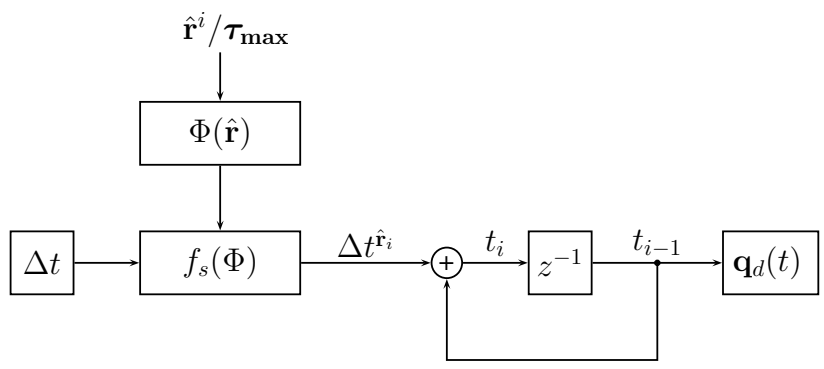

Fig. 4. Block diagram of the time generator in the trajectory scaling. 


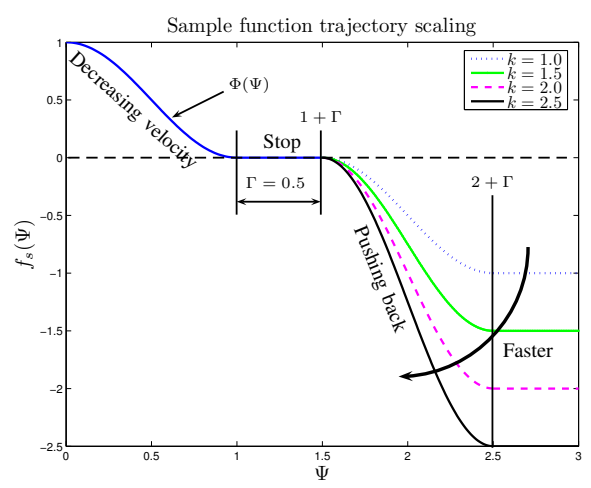

Fig. 5. Scaling function of the time increment.

the estimated external torque was chosen to be

$$
\Psi\left(\hat{\mathbf{r}}_{i}\right)=\frac{1}{\alpha_{\hat{\mathbf{r}}}}\left(\frac{\hat{\mathbf{r}}_{i}}{\tau_{\max }} \cdot \frac{\Delta \mathbf{q}_{\mathbf{d}}^{i}}{\left\|\Delta \mathbf{q}_{\mathbf{d}}^{i}\right\|}\right)_{+}
$$

where $\Delta \mathbf{q}_{\mathbf{d}}{ }^{i}=\mathbf{q}_{\mathbf{d}}{ }^{i+1}-\mathbf{q}_{\mathbf{d}}{ }^{i}$ denotes the difference vector of two consecutive desired via points (e.g. provided by a path planner), $\tau_{\max } \in \Re^{n}$ is the vector of the maximal nominal joint torques specified for the $\operatorname{robot}^{5}$, and "+" denotes the restriction of the term in brackets to positive values for each component. In this way, only external forces pushing against the natural evolution of the trajectory will have an effect on the behavior of the $\operatorname{robot}^{6} \cdot \alpha_{\hat{\mathbf{r}}}$ is a value for adjusting the overall disturbance sensitivity by specifying the normalized collision torque along the trajectory for which the motion should stop, see Fig. 5. The function $f_{s}(\Psi)$ is given by

$$
f_{s}(\Psi)=\left\{\begin{array}{cc}
\Phi(\Psi) & 0 \leq \Psi<1 \\
0 & 1 \leq \Psi \leq 1+\Gamma \\
k \Phi(\Psi-(1+\Gamma))-k & 1+\Gamma<\Psi \leq 2+\Gamma \\
-k & 2+\Gamma<\Psi
\end{array}\right.
$$

where $k \in \Re R^{+}$is a positive factor that determines the decrement velocity. $\Gamma$ is an optional dead-zone. Furthermore, $\Phi($.$) is a monotonically decreasing function$

$$
\Phi:[0,1] \rightarrow[0,1] .
$$

The piecewise defined function $f_{s}(\Psi)$ enables, depending on the disturbance input $\Psi$ to slow down the robot until zero velocity and, after overcoming a dead-zone $\Gamma$, pushing it back along its original path. A sample function for $f_{s}(\Psi)$ is given in Fig. 5. It shows two sinusoidal branches that define the slowing down and back-pushing velocity and an optional dead-zone. The monotonically decreasing function $\Phi$ was implemented as

$$
\Phi(\Psi)=\frac{1}{2}(1+\cos (\pi \Psi))
$$

This function shows better performance than e.g. linear scaling because noise in the detection signal has much less influence on the trajectory scaling in the absence of external torques $(\Psi=0)$. A related approach, but for scaling of rhythmic movements was introduced in [17]. Further related

\footnotetext{
${ }^{5}$ Dividing $\hat{\mathbf{r}}_{i}$ by $\boldsymbol{\tau}_{\max }$ weights external torques according to the specified maximum torque for each joint.

${ }^{6}$ However, one could use the signal as well to accelerate the robot if a human pushes it along its desired trajectory.
}

work can be found in [18], [19]. This reaction strategy was implemented for both the joint and the Cartesian case and was shown on the Automatica $2006 \& 2008$ trade fairs (see also [20], [21]).

\section{A. Combining Trajectory Scaling, Collision Detection \& Reaction}
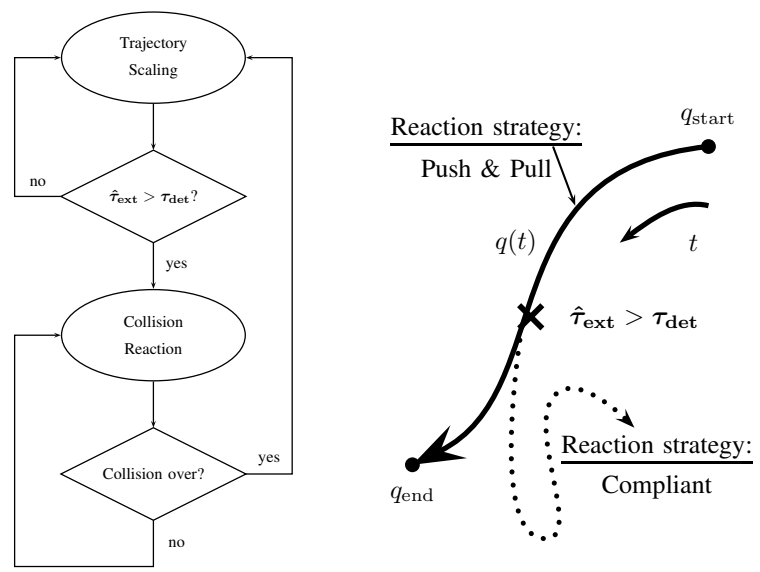

Fig. 6. Combining trajectory scaling and other reaction strategies based on the magnitude of the disturbance signal. As long as the torque estimation remains within a certain limit band, trajectory scaling is active. In case this threshold is exceeded the robot switches to one of the other reaction schemes.

Typically, undesired impacts are characterized by high peak forces resulting in high joint torques. Therefore, a very easy way to distinguish between desired interaction and accidental collisions is to use the magnitude of $\hat{\mathbf{r}}$ (or any other estimation $\hat{\tau}_{\text {ext }}$ of the external torques). Trajectory scaling ensures that during normal operation mode only a certain maximum static force (depending on $f_{s}$ ) can act on a human. If he/she pushes harder, the robot moves back along $\mathbf{q}_{\mathbf{d}}$ and as soon as the pushing force is too high $\left(\|\hat{\mathbf{r}}\| \geq r_{\max }^{\text {switch }} \in \Re^{+}\right)$, the robot switches to one of the other reaction Strategies 1-4 (e.g. in case of Strategy 2 the robot poses due to its compliance no threat anymore), see Fig. 6. Thus, a combination of reaction strategies performs an intuitive and effective response to desired physical interaction or unintended collision/clamping. In Fig. 7 this combined reactive strategy is visualized.

\section{EXPERIMENTAL RESULTS}

In this section we will show the benefits gained from the use of the collision detection and reaction algorithms by evaluating impact tests with the LWRIII on different human body parts, a dummy, and an especially built test-bed.

\section{A. Collision Detection and Reaction Results for the Neck}

The first results are related to the neck force during a collision with a HybridIII-dummy ${ }^{7}$. The experimental setup and the neck force $F_{\text {res }}^{\text {Neck }}$ caused by head impacts for a robot velocity of $0.2 \mathrm{~m} / \mathrm{s}$ are illustrated in Fig. 8 (right). The actual impact is characterized by a very short peak, which duration and maximum value depend on the impact velocity. After this impact phase, a steadily growing neck bending force arises in absence of a collision detection. The plot with

\footnotetext{
${ }^{7}$ The results with the HybridIII-dummy were presented in [1] and are shown here to support the effectiveness of the collision detection and reaction at standardized crashtest facilities.
} 

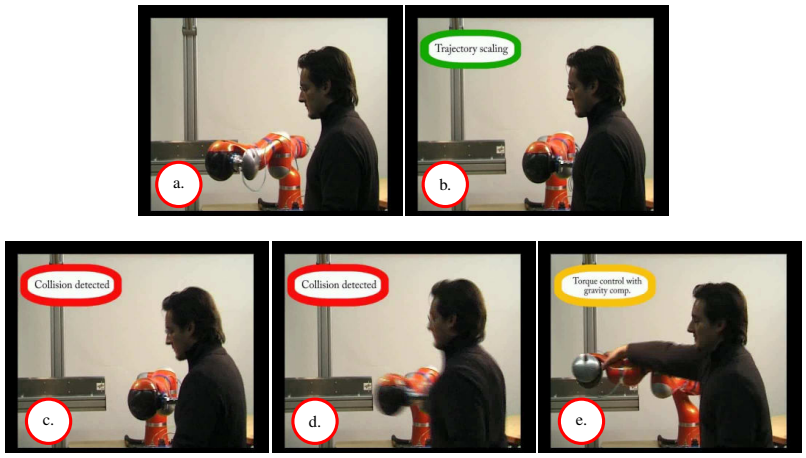

Fig. 7. Combining safety during the execution of a task and during a real collision: (a.) The robot moves position controlled along its desired trajectory. (b.) The robot slows down (trajectory scaling) and in the end stops after physical contact with the human. If the human would step aside the robot would continue to move along its desired trajectory. (c.) The human pushes harder against the robot and consequently the collision detection is triggered (d.) The robot compliantly floats away in torque control with gravitation compensation (Strategy 2). (e.) Now, the robot can easily be moved around without being able to cause any harm.
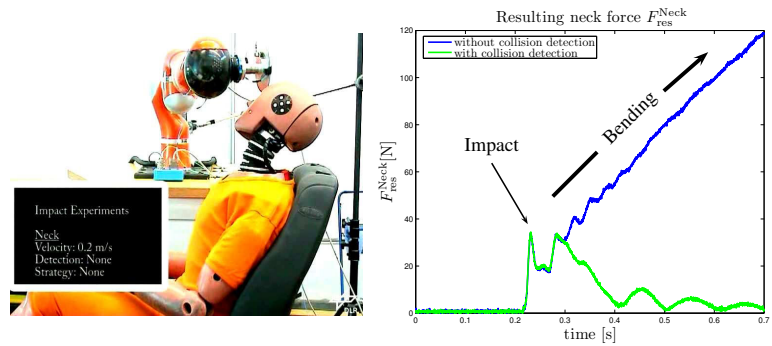

Fig. 8. Bending the dummy neck at a robot velocity of $0.2 \mathrm{~m} / \mathrm{s}$ (left) Resulting dummy neck force with and without collision detection and strategy 2 (right).

activated collision detection clearly shows the reduction in neck force due to the collision reaction strategy ${ }^{8}$. In case of a constrained human as depicted in Fig. 8 (left) one is therefore able to reduce the neck forces far below their critical value of $1.1 \mathrm{kN}$ in any direction [1], [22].

\section{B. Human Arm Measurements \& Collision Test-bed}

In order to objectively compare collision reaction strategies and since the arms of crash-test dummies are not equipped with appropriate sensors, a simple collision testbed was built up to emulate robot-human arm impacts. This is a $1 \mathrm{DOF}$ mechanism with adjustable impedance, of which a spring stiffness and a mass can be adapted to fit with impact characteristics of interest, see Fig. 9 (upper). We have mimicked the impact behavior of the human arm in a typical impact configuration, the one shown in Fig. 11 (right), and used it as a basis for comparing the presented reaction strategies. Indeed, we are aware of the problems in fitting a certain model to a human arm that is potentially nonlinear and of higher order. Furthermore, one could argue that the human reacts with an impedance response to the impact. However, at this point we intended to replicate only the rough behavior of the human arm for a specific situation in order to give a common ground for comparing impact reaction strategies on a fair basis. It was not our intention to construct an anthropomorphic model of the human arm in this work.

\footnotetext{
${ }^{8}$ This experiment is shown as well in the attached video.
}
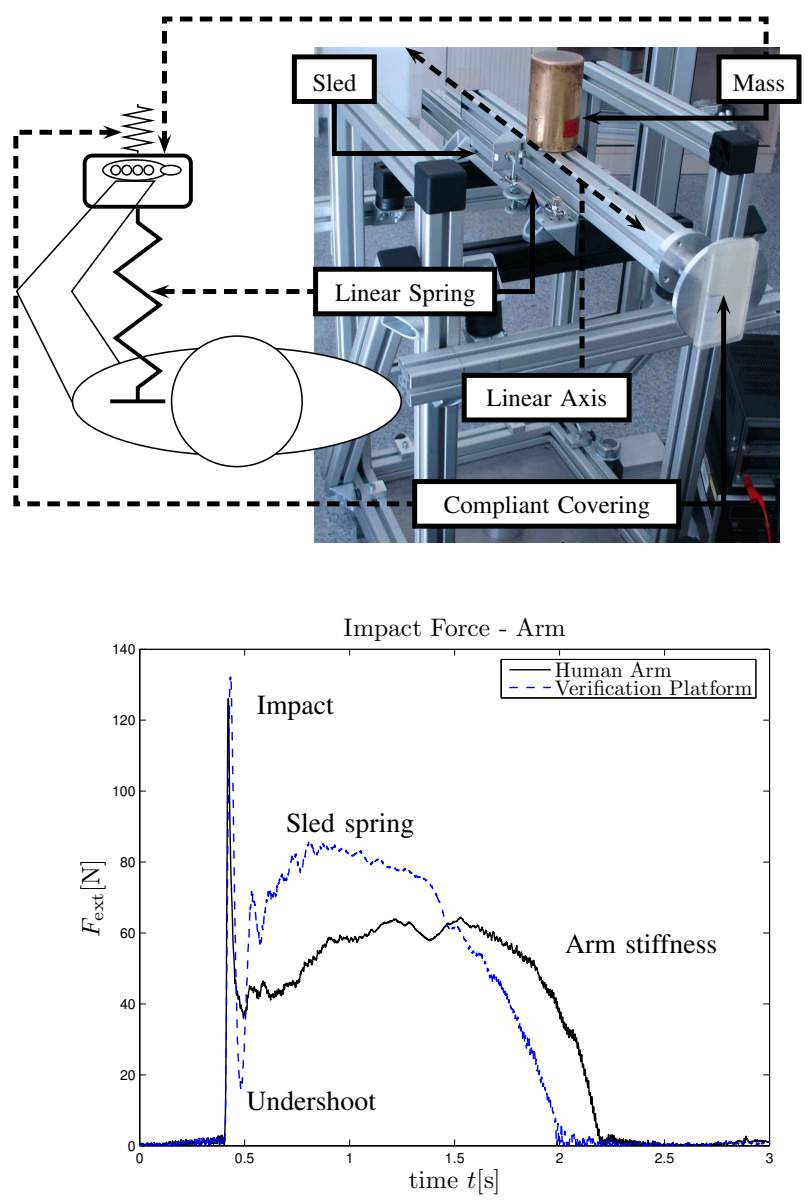

Fig. 9. Collision test-bed (upper), representing a simplified model of the human arm (upper), and the resulting impact forces for a human arm and the collision test-bed at a robot velocity of $0.4 \mathrm{~m} / \mathrm{s}$ (lower). After an initial peak a stiffness profile is observed, representing the human response in this particular experiment.

The force occurring during a typical arm impact is shown in Fig. 9 (lower) for the reconfiguration trajectory from "elbow up" to "elbow down". The robot was used to measure contact forces $^{9}$, kinematic configurations, and velocities. In comparing the test-bed with the human, some differences in the impact characteristics can be observed. Especially the damping in the collision test-bed is considerably lower, showing an undershoot after the consistent impact force. To somewhat overcome this deficit, the sled spring was pretensioned, leading to a biased spring and thus to higher forces during the bending process. For future collision tests the test-bed will be extended by a mechanical damping element to optimize the impact behavior of the sled.

\section{Comparison of Collision Reaction Strategies with the Test-bed}

The results for impacting the LWRIII against the collision test-bed with various reaction schemes are shown in Fig. 10. From the instant of impact on, the contact force (upper) and the Cartesian displacement (lower) are shown. Furthermore, on the lower plot the collision detection signal is also reported, indicating how fast the robot actually reacts as

${ }^{9}$ In all experiments the contact force was measured with a JR3 force/torque sensor. 

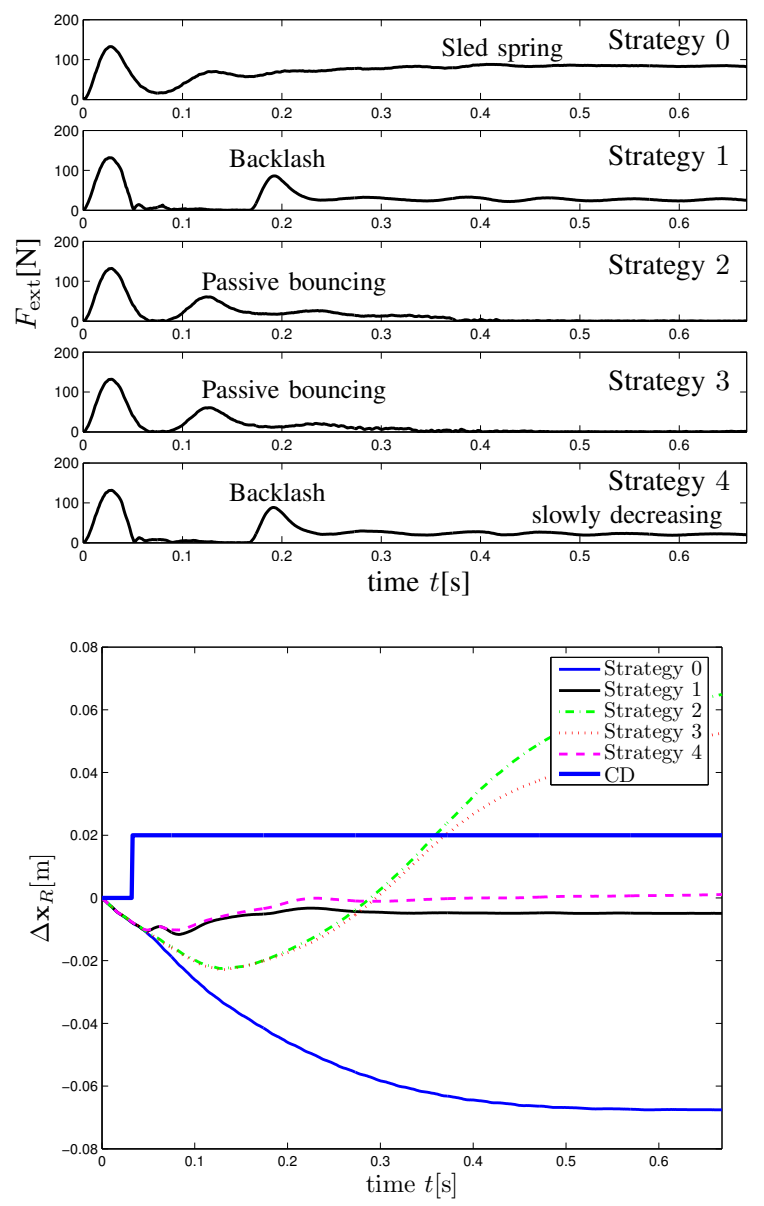

Fig. 10. Comparison of different collision reaction strategies with the test-bed at an impact velocity of $0.4 \mathrm{~m} / \mathrm{s}$. The point of origin with $t=0$ indicates the instant of impact. Apparently, the maximum initial peak force, which is passed after less than $25 \mathrm{~ms}$, cannot be reduced for the impact with the test-bed. Although for Strategy 2 and 3 no backlash can be observed, a second "impact" occurs. This is a further bending of the sled spring due Strategy 0 , but due to the compliant behavior, in a very alleviated from.

soon as a collision is detected. Here, trajectory scaling was not evaluated on purpose, since it is intended to serve as a feature during task execution to allow interaction and not as a collision reaction scheme which shall only be activated during high load impacts. Strategy 1 and 4 show very fast reaction after the first force peak and then lose contact with the accelerated sled. Due to the backlash of the sled a second impact occurs in both cases. As one can see, Strategy 4 seems to be the fastest to withdraw from the external force in the first $200 \mathrm{~ms}$. Unfortunately, it could not be tuned such that the slowly decreasing contact force after the backlash is eliminated. This drawback is probably caused by the time delay in the admittance control loop and the higher Coulomb friction of the robot compared to the one used in [10]. However, the maximum displacement for both strategies is $\approx 10-12 \mathrm{~mm}$, showing a much faster reduction than Strategies 2 and 3. Additionally, the influence of the test-bed spring is entirely canceled.

In general, Strategies 2 and 3 show very similar behavior, leading to the conclusion that the additional inertia shaping (Strategy 3) is not significantly contributing to an improvement in reaction behavior. Apart from that, these two strategies do not lose contact as abruptly as Strategies 1 and $4 \mathrm{do}$, but the contact force reduces after $<400 \mathrm{~ms}$ to zero due to the very convenient compliant behavior. These observations lead to the recommendation to combine the speed of Strategy 4 to avoid the higher displacement and entire influence of the sled spring, with the convenient compliant behavior of Strategy 2 by subsequently switching to this mode.

\section{Real Collisions with the Human Arm \& Chest}

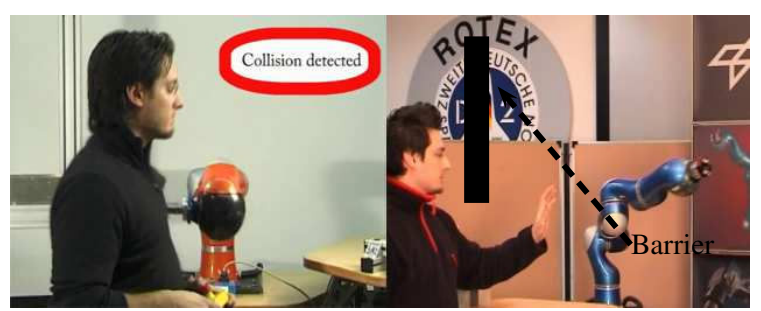

Fig. 11. Real collisions with the chest and arm were conducted up to a robot velocity of $2.7 \mathrm{~m} / \mathrm{s}$.

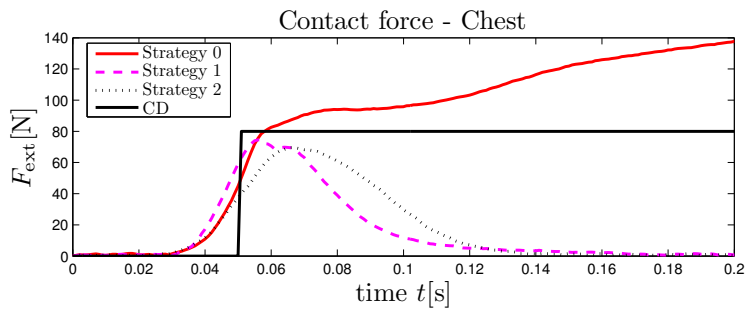

Contact force - Arm

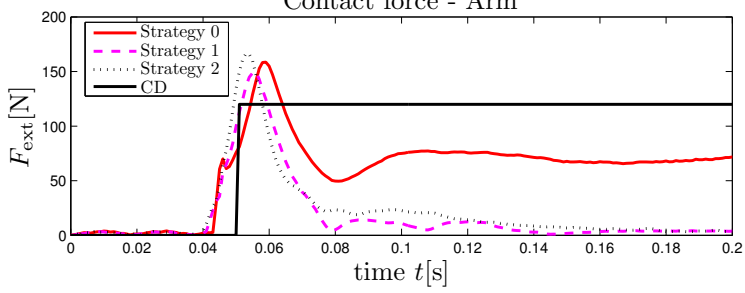

Fig. 12. Resulting contact force with and without collision detection and reaction strategy for the human chest (upper) at impact velocities of $0.7 \mathrm{~m} / \mathrm{s}$ and the arm (lower) at $1.5 \mathrm{~m} / \mathrm{s}$. These tests were carried out up to an impact velocity of $2.7 \mathrm{~m} / \mathrm{s}$ but at such impact velocities it is very hard to reproduce testing conditions accurately enough.

During the extensive tests with the crash-test dummy and the collision test-bed, the collision detection proved to work very reliably and was effective in reducing the forces far below any level which is dangerous to humans. It turned out that indeed a human can stop the robot with the hand at surprisingly moderate forces. In contrast to the collisions with the test-bed, even forces occurring during an impact with the human chest were significantly reduced by the collision detection and reaction ${ }^{10}$. In order to show the effectiveness of the collision detection mechanisms, real impact tests were conducted with a non-clamped human chest and an outstretched arm. The human stood relaxed and was not able to see the robot coming. In Fig. 11 the impact positions are shown. The one for the human arm was chosen such that it is in a comfortable configuration and not prestressed. Since for these tests a difference in the contact forces for the compliant

${ }^{10}$ Due to the much higher inertia and therefore significantly lower acceleration of the upper trunk, contact with the human is not rapidly lost and thus reaction strategies can limit forces acting on the chest. 
reaction strategies is not measurable due to the large variation caused by the human ${ }^{11}$, we focussed on the first three ones, namely Strategies $0,1,2$. For the chest impacts the detection activates within $\approx 14 \mathrm{~ms}$, bringing the contact force down to zero within $<100 \mathrm{~ms}$ and limiting it below $\approx 75 \mathrm{~N}$ for both the active strategies that were evaluated, see Fig. 12 (upper). For Strategy 1 the human is accelerated fast enough due to the impact force and thus loses contact in case the robot abruptly stops. Generally, even without collision detection and reaction the impact forces can be kept far below the tolerance force $F_{\text {ext }}^{x, \text { tol }} \in[1.15 \div 1.7] \mathrm{kN}$ of the chest [23]. Furthermore, the collision reaction keeps the contact forces far below the proposed value of $150 \mathrm{~N}$ in ISO-10218 [24] which would be exceeded for chest impacts with Strategy 0. The contact force for the arm is illustrated in Fig. 12 (lower), showing a somewhat different behavior. After a short impact, which cannot be prevented or attenuated by the collision detection and reaction, the impact forces reduce to zero for Strategy 1 and 2. For Strategy 0, another safety feature of the LWRIII activates because of the increasing contact force. In fact, a low-level stop is triggered by the exceedance of the measured joint torque. For the human arm very limited biomechanical tolerance data is available. We finally note that the $150 \mathrm{~N}$ proposed by ISO-10218 are from our point of view too conservative for blunt impacts due to the fact that a $50 \%$ risk of elbow fracture corresponds to forces as large as $1780 \mathrm{~N}$ [25].

\section{E. Trajectory Scaling}

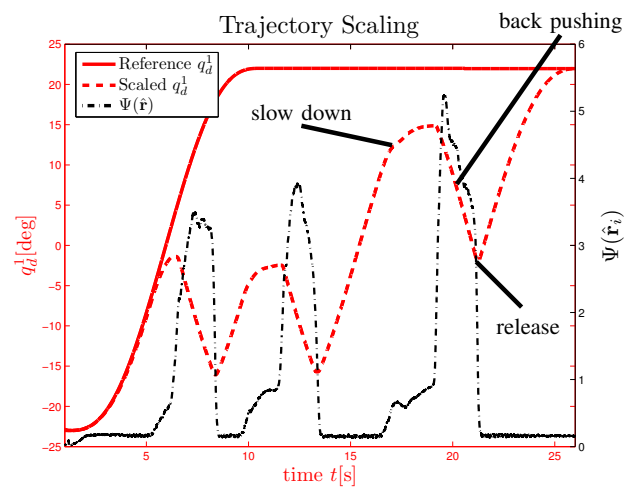

Fig. 13. Measured trajectory scaling for a sample trajectory implemented on the LWRIII. The left $y$-axis is relevant for the reference and scaled joint angle $q_{d}^{1}$.

Experimental results for the trajectory scaling are presented in Fig. 13. A reference trajectory $q_{d}^{1}$ for the first joint (solid line) is given for nominal free motion. It is a 5 th order polynomial from $q_{d, \text { start }}^{1}=-23^{\circ}$ to $q_{d \text {,end }}^{1}=22^{\circ}$. During the execution of this trajectory the human pushes against the robot $^{12}$ and the resulting scaled desired position $q_{d}^{1}$ (dashed line) shows the slowing down and back-pushing along the trajectory depending on the disturbance input $\Psi(\hat{\mathbf{r}})$ (dasheddotted line).

We remark that trajectory scaling is intended for continuous physical interaction without switching the control mode. It is used when the robot is position controlled, leading to a very convenient way to interact with the robot without forcing a global change of its behavior. Still, the user has the possibility to almost instantaneously stop the robot by pushing against it. A major advantage of trajectory scaling

\footnotetext{
${ }^{11}$ This is one important reason why the collision test-bed was built.

${ }^{12}$ See the attached video.
}

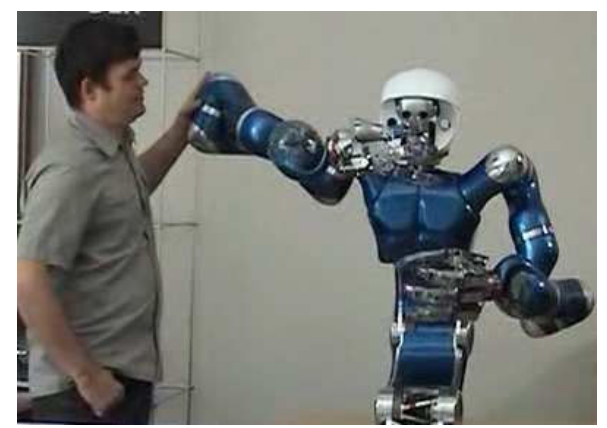

Fig. 14. Physical interaction with the DLR humanoid Justin

is that for a complex robot, such as the DLR dual-arm humanoid Justin, one has only to slow down one of the sub-robots it consists of. For instance, pushing against one of the elbows as shown in Fig. 14 slows down and finally stops/reverts both arms, both hands, the torso, and the neck and not only the touched arm ${ }^{13}$.

\section{F. Collision Detection for Robot Protection}

Apart from ensuring safety to the human by the presented algorithms we observed that the impact is posing a risk to the robot hardware as well. An observation, already made at an impact velocity of $1 \mathrm{~m} / \mathrm{s}$ during the impact tests with the HybridIII-dummy [1], is that the specified maximum joint torques of the robot were exceeded for several milliseconds during the impact ${ }^{14}$. This shows that the robot is exposed to enormous loads during such contacts and countermeasures are needed for ensuring safety of the robot. Of course speed limitation to subcritical values is one option but at the same time it severely limits the performance of the robot. Another solution seems to be a joint stiffness reduction, see [26], [1]. However, a further alternative to protect the robot to a certain extent are the fast collision detection \& reaction strategies presented in this paper.

Fig. 15 shows the measured joint torque in the 1st and 4th axis during chest (a.) and head (b.) impacts at $2 \mathrm{~m} / \mathrm{s}$. Clearly the peak joint torque can be significantly reduced by switching the control mode as soon as a collision is detected for the chest.

A different behavior was observed for the head. While the first joint is still able to reduce its peak torque, for the fourth one this is no longer the case presumably due to the lower inertia.

\section{CONCLUSION}

In this paper two collision detection and several reaction strategies were presented and extensively validated by experiments. The robot is able to detect and distinguish unexpected collisions from an intended cooperation, in which a human stretching out his arm, tries to catch the robot. This provides a subjective "safe" feeling. The robot inflicted no harm to the operator at any of the considered velocities and it was always possible to detect the collision and let the robot switch to one of the investigated reaction strategies. The human was never in danger even for impacts with the human chest at robot velocities up to $2.7 \mathrm{~m} / \mathrm{s}$. Especially collision reaction Strategies 2 and 3 act very pleasing and let the operator feel

\footnotetext{
${ }^{13}$ At this point we assume one common time basis for all parts of the robot. This experiment can be viewed in the attached video.

${ }^{14}$ In the robot a mechanical stop limits the deflection range of the torque sensor which then goes into saturation. A low-level emergency stop is initialized as soon as this event is triggered.
} 

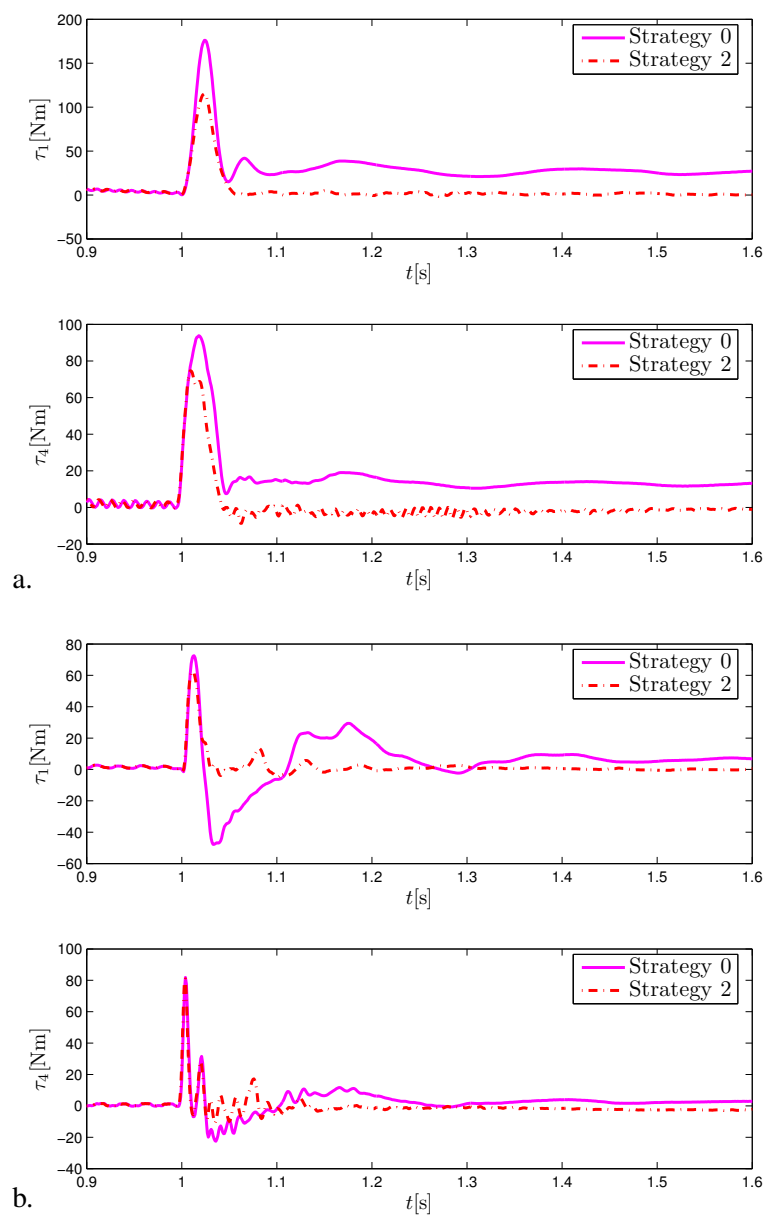

Fig. 15. Joint torques in the first and fourth joint for impacts at $2.0 \mathrm{~m} / \mathrm{s}$ with a HybridIII-dummy chest (a.) and head (b.). The used collision reaction scheme was Strategy 2. For the chest it was possible to decrease the collision peak torque for both joints. For the head only a marginal attenuation of the joint torques is achieved.

that he has full control of the robot. The ability to actively stop the execution via trajectory scaling is very convenient and incorporates reactive behavior with a robot in a position control loop. Furthermore, also the benefit from the robot's side was exemplified, consisting of the protection of the joints from high external forces.

Future work will focus on the extension and enhancement of the trajectory scaling beyond pushing the robot back and forth along its desired path, so as to find an adaptive trajectory deformation. This shall allow the robot to avoid collision sources and float compliantly away, while finding its way back to the desired trajectory once the collision is over. Finally, in the presence of redundancy, a meaningful way to combine Cartesian trajectory scaling with null-space movements, has still to be found.

\section{REFERENCES}

[1] S. Haddadin, A. Albu-Schäffer, and G. Hirzinger, "Safety Evaluation of Physical Human-Robot Interaction via Crash-Testing," in Robotics: Science and Systems Conference 2007 (RSS2007), Atlanta, USA, 2007.

[2] S. Oberer and R. D. Schraft, "Robot-Dummy Crash Tests for Robot Safety Assessment," in IEEE Int. Conf. on Robotics and Automation (ICRA2007), Rome, Italy, 2007, pp. 2934-2939.
[3] M. Zinn, O. Khatib, B. Roth, and J.K.Salisbury, "Playing It Safe Human-Friendly Robots," IEEE R\&A Mag., vol. 11, pp. 12-21, 2002.

[4] A. Bicchi and G. Tonietti, "Fast and Soft Arm Tactics: Dealing with the Safety-Performance Trade-Off in Robot Arms Design and Control,' IEEE R\&A Mag., vol. 11, pp. 22-33, 2004

[5] K. Ikuta, H. Ishii, and M. Nokata, "Safety Evaluation Method of Design and Control for Human-Care Robots," Int. J. of Robotics Research, vol. 22, no. 5, pp. 281-298, 2003.

[6] J. Heinzmann and A. Zelinsky, "Quantitative Safety Guarantees for Physical Human-Robot Interaction," Int. J. of Robotics Research, vol. 22, no. 7-8, pp. 479-504, 2003.

[7] H.-O. Lim and K. Tanie, "Human Safety Mechanisms of HumanFriendly Robots: Passive Viscoelastic Trunk and Passively Movable Base," Int. J. of Robotics Research, vol. 19, no. 4, pp. 307-335, 2000.

[8] M. Wassink and S. Stramigioli, "Towards a Novel Safety Norm for Domestic Robots," in IEEE/RSJ Int. Conf. on Intelligent Robots and Systems (IROS2007), San Diego, USA, 2007, pp. 3354-3359.

[9] G. Hirzinger, N. Sporer, A. Albu-Schäffer, R. Krenn, A. Pascucci, and M. Schedl, “DLR's Torque-Controlled Light Weight Robot III - Are We Reaching The Technological Limits now?" in IEEE Int. Conf. on Robotics and Automation (ICRA2002), Washington, DC, USA, 2002, pp. $1710-1716$

[10] A. De Luca, A. Albu-Schäffer, S. Haddadin, and G. Hirzinger, "Collision Detection and Safe Reaction with the DLR-III Lightweight Manipulator Arm," in IEEE/RSJ Int. Conf. on Intelligent Robots and Systems 2006 (IROS2006), Beijing, China, 2006, pp. 1623-1630.

[11] A. Albu-Schäffer, S. Haddadin, C. Ott, A. Stemmer, T. Wimböck, and G. Hirzinger, "The DLR Lightweight Robot Lightweight Design and Soft Robotics Control Concepts for Robots in Human Environments," Industrial Robot Journal, vol. 34, pp. 376-385, 2007.

[12] M. Spong, "Modeling and Control of Elastic Joint Robots," IEEE Journal of Robotics and Automation, vol. 109, pp. 310-319, 1987.

[13] A. Albu-Schäffer, C. Ott, and G. Hirzinger, "A Unified Passivity-based Control Framework for Position, Torque and Impedance Control of Flexible Joint Robots," Int. J. of Robotics Research, vol. 26, pp. 2339, 2007.

[14] A. De Luca and R. Mattone, "An Adapt-and-Detect Actuator FDI Scheme for Robot Manipulators," in IEEE Int. Conf. on Robotics and Automation 2004 (ICRA2004), New Orleans, USA, 2004, pp. 49754980.

[15] _ "Sensorless Robot Collision Detection and Hybrid Force/Motion Control," in IEEE Int. Conf. on Robotics and Automation 2005 (ICRA2005), Barcelona, Spain, 2005, pp. 1011-1016.

[16] A. Albu-Schäffer and G. Hirzinger, "Cartesian Impedance Control Techniques for Torque Controlled Light-Weight Robots," in IEEE Int. Conf. on Robotics and Automation (ICRA2002), Washington, DC, USA, 2002, pp. 657-663.

[17] H. Urbanek, A. Albu-Schäffer, and P. van der Smagt, "Learning from demonstration: repetitive movements for autonomous service robotics," in IEEE/RSJ Int. Conf. on Intelligent Robots and Systems (IROS2004), Sendai, Japan, 2004, pp. 3495-3500.

[18] P. Li and R. Horowitz, "Passive velocity field control of mechanical manipulators," in IEEE Int. Conf. on Robotics and Automation (ICRA1995), Nagoya/Aichi,Japan, 1995, pp. 2764-2770.

[19] T.-J. Tarn, N. Xi, and A. K. Bejczy, "Path-based approach to integrated planning and control for robotic systems," Automatica, vol. 32, no. 12 pp. 1675-1687, 1996.

[20] S. Haddadin, A. Albu-Schäffer, and G. Hirzinger, "Approaching Asimov's 1st Law," HRI Caught on Film, 2nd ACM/IEEE Int. Conf. on Human-Robot Interaction (HRI2007), Washington DC, USA, pp. 177$184,2007$.

[21] C. Borst, C Ott, T Wimböck, B Brunner, F Zacharias, B. Bäuml, U. Hillenbrand, S. Haddadin, A. Albu-Schäffer, and G. Hirzinger, "A Humanoid Upper Body System for Two-Handed Manipulation," IEEE Int. Conf. on Robotics and Automation (ICRA2007), Rome, Italy, pp 2766-2767, 2007.

[22] EuroNCAP, "European Protocol New Assessment Programme - Assessment Protocol and Biomechanical Limits," 2003.

[23] L. Patrick, "Impact Force Deflection of the Human Thorax," SAE Paper No.811014, Proc. 25th Stapp Car Crash Conference, pp. 471496, 1981.

[24] ISO10218, "Robots for industrial environments - Safety requirements - Part 1: Robot," 2006

[25] S. Duma, B. Boggess, J. Crandall, and C. MacMahon, "Fracture tolerance of the small female elbow joint in compression: the effect of load angle relative to the long axis of the forearm," Stapp Crash Journal, vol. 46, pp. 195-210, 2002.

[26] S. Haddadin, T. Laue, U. Frese, and G. Hirzinger, "Foul 2050: Thoughts on Physical Interaction in Human-Robot Soccer," in IEEE/RSJ International Conference on Intelligent Robots and Systems (IROS2007), San Diego, USA, pp. 3243-3250. 